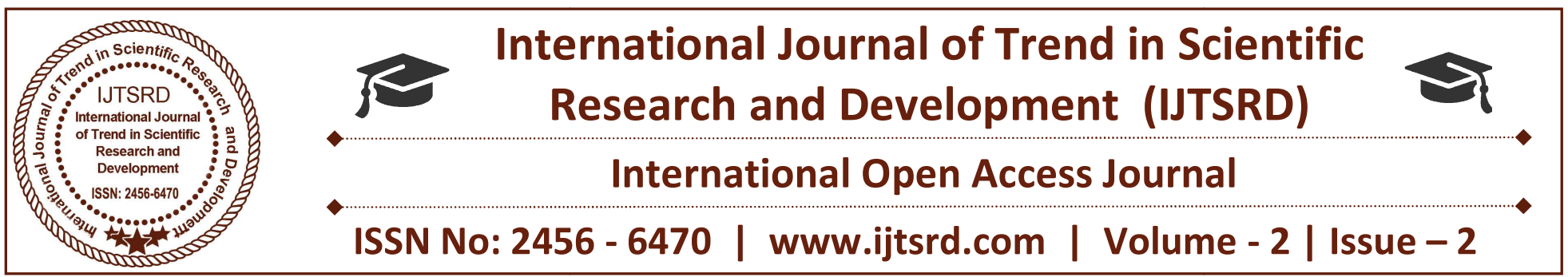

\title{
Is the Sum of Interior Angles of a Triangle Always $180^{\circ}$ ?
}

\author{
Swachhatoya Ghosal \\ Student, Department of Education, Vinaya Bhavana, \\ Visva Bharati University, West Bengal, India
}

\author{
Vinaya Bhavana \\ Department of Education, Vinaya Bhavana, \\ Visva Bharati University, West Bengal, India
}

\section{ABSTRACT}

In $19^{\text {th }}$ century we have the concept of Non Euclidean geometry that is divided into two parts spherical geometry and hyperbolic geometry. Euclidean plane is not quite the same as the Cartesian plane. In geometry a plane is a flat, two dimensional surface that extends infinitely far but has no thickness. In reality it is bit difficult to visualise or think a plane. The actual concept of plane is imaginary on the surface of the earth. In broader sense it is no longer true that the sum of a triangle is $180^{\circ}$, however to some extent it may be true for very small triangle because from the perspective of a very small triangle, the surface of a sphere is nearly flat otherwise it is pertinent to consider for a spherical triangle (A spherical triangle formed by the arcs of the three great circles each of whose poles is the vertex of a given spherical triangle) or hyperbolic triangle (A hyperbolic triangle is just three points connected by the hyperbolic line segment) the sum of the three interior angles is less than or more than $180^{\circ}$ respectively.

Keywords: Euclidean plane, Cartesian plane, Triangle, Sphere, and Hyperbola.

\section{INTRODUCTION}

Euclid geometry is based on Euclid axioms which were given in the book namely "The Elements". This book was written by Euclid in about 300 B.C. Girolamo Saccheri (1667-1733), a Jewish professor of mathematics at the University of Pavia, Italy, showed that the Euclid's fifth postulate is not true and has given several theorems of Non-Euclidean Geometry. Presently we have the concept about Euclidean and Non Euclidean geometry. Besides many others Non
Euclidean geometry is basically spherical and hyperbolic geometry.

In plane geometry, basic concepts are points and straight lines where geodesics are the shortest path between points and angles are defined by straight lines. But in spherical geometry a spherical triangle (A triangle whose sides are minor arcs of three great circles) is based on two dimensional surface of a sphere where geodesics are the great circle and angles are defined by great circle. In hyperbolic geometry a hyperbolic triangle (A triangle with just three points connected by hyperbolic line segments) whose three vertices of hyperbolic space of an arbitrary dimension always lie on the same plane. Accordingly both spherical triangle and hyperbolic triangle violates the Euclid's fifth postulates means there is no point through which a line can be drawn that never intersects a given line, in broader sense a statement that is equivalent to parallel postulate is that there exists a triangle whose angle add up to $180^{\circ}$.

Thus Geometry is one of the oldest wings of mathematics, concerns with day to day life. The name geometry has come up from two or three point. In Greek word Geo means earth and Metron means measure, meaning 'measurement of the earth'. It is concerned with shape, special relationship and properties of surrounding objects. Geometry is not limited to flat surfaces and three dimensional objects; rather it can be used for most abstract thoughts and images as well. As now in the ancient age also people used geometry in day to day life. 
During the excavations at Harappa and Mohenjo-Daro it showed that the Indus Valley Civilisation (about 3000 BCE) made extensive use of geometry as it was a highly organized society with proper roads, drainage system, and storage system of grains. The houses had many rooms of different types. The bricks used for constructions were kiln fired and the ratio length: breadth : thickness, of the bricks was found to be $4: 2$ : 1. In ancient India, the Sulbasutras (800 BCE to 500 $\mathrm{BCE}$ ) were the manuals of geometrical constructions. The geometry of the Vedic period originated with the construction of altars (or vedis) and fireplaces for performing Vedic rites. Instructions regarding the fire place and their shapes and areas were clearly mentioned along with that square and circular altars were used for household rituals, while altars whose shapes were combinations of rectangles, triangles and trapeziums were required for public worship. Though accurate geometric methods were used for the constructions of altars, the principles behind them were not discussed. And the knowledge of method and modification was happening through either orally or through palm leaf messages, or by other ways. Use of Geometry is also seen the civilizations of Babylonia. The use of Geometry in Babylonia and Egypt was purely practical oriented which is similar to India and Rome. All of the mentioned civilization have tried very little to develop it as a systematic science which is not same in case of civilisations of Greece. At that time Euclid, a teacher of mathematics at Alexandria in Egypt, collected all the known work and arranged it in his famous treatise, called 'Elements'. He divided the 'Elements' into thirteen chapters, each called a book. These books influenced the whole world's understanding of geometry for generations to come. He is known as the Father of Geometry.

Euclid propounded the foundation of scientific and systematic Geometry and while doing so he made a point to the mass that mathematical statements need to be proved. According to him that though a statement might sound like truth but still it will have the chance to be wrong and thus the only way to be certain is to give a proof. To provide proof there is a need to start it somewhere and proving so Euclid made the amazing step of realizing that mathematics needs axioms which is an agreed starting point which does not require proof.

Euclid stated that let us agree on some basic 'facts', and let us also agree that from then on everything else must be proved. With this in mind he then laid down the axioms of what we now call Euclidean geometry, and then went on to develop this geometry to a very high level indeed. Euclid geometry is based on Euclid axioms which were given in the book namely "The Elements". This book was written by Euclid in about 300 B.C.

Girolamo Saccheri (1667-1733), a Jewish professor of mathematics at the University of Pavia, Italy, showed that the Euclid's fifth postulate is not true and has given several theorems of Non-Euclidean Geometry.

\section{OBJECTIVES OF THE STUDY}

i) to promote critical thinking and reasoning abilities in new and novel ideas of Euclidean and NonEuclidean geometry.

ii) to promote critical thinking and reasoning abilities in new and novel ideas of Euclidean plane, spherical geometry, and hyperbolic geometry.

iii) to develop and expand thinking about angle sum property of spherical triangle and hyperbolic triangle.

iv) to modify the orthodox ideas about the triangle(s) and their angle sum property.

\section{BODY OF THE WORK}

From the introduction -we get to know about the history of geometry and the journey of forming the concept of Euclidean geometry to Non Euclidean geometry.

\section{Euclidean triangle:}

In Euclidean geometry it is learnt from angle sum property, for any triangle the sum of the interior angles would be always $180^{\circ}$.

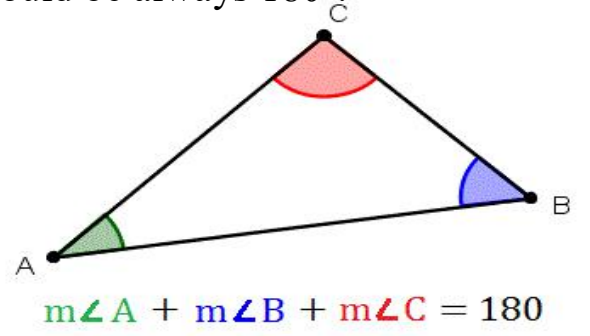

Now we know at present geometry is divided into two parts, Euclidean geometry and non Euclidean geometry. There are two main types of nonEuclidean geometry. The first one is spherical surfaces, dealing with spherical surface. And the 
second one is hyperbolic geometry, dealing with the geometry of saddle-shaped surfaces.

\section{Spherical triangle:}

The sum of the angles of a triangle on a sphere is $180^{\circ}(1+4 \mathrm{f})$, where ' $\mathrm{f}$ is the fraction of the sphere's surface that is enclosed by the triangle. For any positive value of ' $\mathrm{f}$ ' this exceeds $180^{\circ}$, and that ranges from $180^{\circ}$ to $540^{\circ}$.

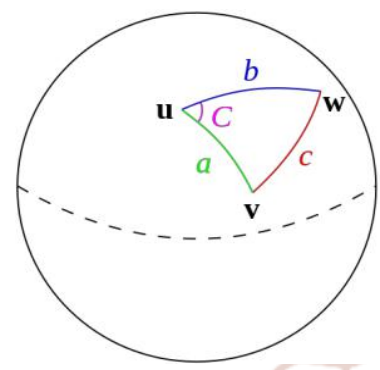

Similarly hyperbolic geometry also consists of various shapes made up of curved lines. Hyperbolic triangle is a triangle formed by three curved lines.

The projection of the spherical triangle would be like this figure

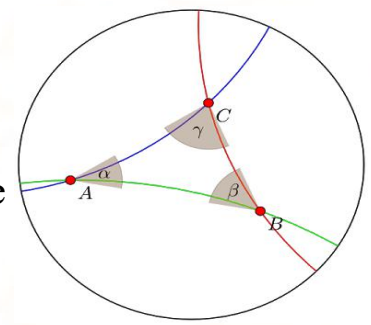

Hence it can be concluded that sum of all the three interior angles of a triangle is always not $180^{\circ}$. For spherical triangle the sum is more than $180^{\circ}$ and for hyperbolic triangle the sum is less than $180^{\circ}$.

The projection of the spherical triangle would be like this figure

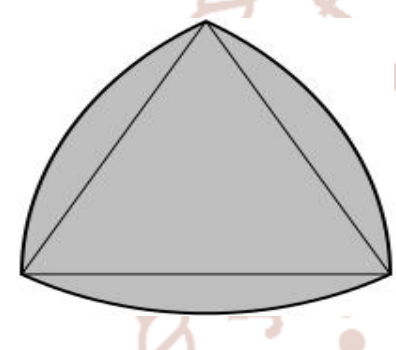

In real life situation watermelon is a sphere. This

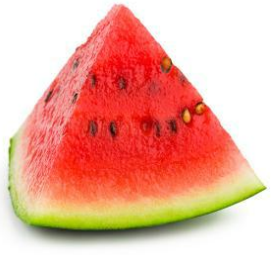
triangle is cut from that and that is $\mathrm{N}$ the shape of a spherical triangle (Upper green lair).

\section{Hyperbolic triangle:}

Every hyperbolic triangle has an inscribe circle but not every hyperbolic triangle has a circumscribe circle, this is the cause when at least one of its vertices is a ideal point or when all its vertices lay on an horocycle or on a one sided hyper-

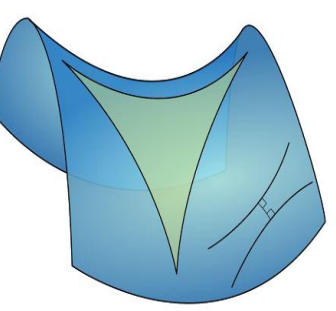

\section{REFERENCE}

1) Beardon, Alan.( 2001January, 2011February). How Much Geometry Are There?

Retrieved from https://nrich.maths.org/1386

2) Beardon,Toni.( 2002 May, 2007June, 2011February ). When the Angles of a Triangle Don't Add up to 180 Degrees : nrich ...retrieved from https://nrich.maths.org/1434

3) Heilbron,J.L .geometry / mathematics | Britannica.com. retrieved from https://www.britannica.com/topic/geometry

4) Introduction to euclid's geometry - ncert. retrieved from ncert.nic.in/ncert/l/iemh105.pdf.

5) O'Dea,Steve.(Dec 17.2016). What are the uses of triangles in our daily life -

6) Quora. retrieved from https://www.quora.com/What-are-the-uses-oftriangles-in-our-daily-life

7) Sing,Hukum. On Euclidean and Non-Euclidean Geometry .retrieved from www.ncert.nic.in/pdf_files/Hukum\%20Singh.pdf 